\title{
Performance Analysis of Spectrum Sharing Systems with Distributed CDD
}

\author{
Kyeong Jin Kim, Hongwu Liu, Marco Di Renzo, and H. Vincent Poor
}

\begin{abstract}
In this paper, a cooperative spectrum sharing system is investigated. To effectually access the radio spectrum licensed to the primary users, the distributed cyclic delay diversity (dCDD) scheme is employed as the transmit diversity scheme for distributed cyclic-prefixed single carrier transmissions. As a set up for the secondary users' network, it is assumed that it is comprising the control unit and a group of remote radio heads. In the secondary users' network, a single primary user transmitter is assumed to be located isotropically, so that a mixture of line-of-sight and non-line-of-sight paths over frequency selective fading channels is considered in this paper. One of the objectives of this paper is to investigate the effects of this new channel model on the outage probability probability promised by dCDD. The closed-form expression for the outage probability is derived first, and then its asymptotic expression is derived to draw the maximum achievable diversity gain. Link-level simulations are also conducted to verify the performance analysis.
\end{abstract}

Index Terms-Distributed CDD, spectrum sharing, cyclicprefixed single carrier transmission, outage probability, diversity gain.

\section{INTRODUCTION}

Maximum ratio transmission (MRT) was proposed as a transmit diversity scheme by [1] for a transmitter equipped with multiple antennas. Recently, MRT has been applied to the distributed system [2]. However, acquiring exact channel state information (CSI) at the transmitter side is a challenging task in distributed wireless communications systems which apply the transmit diversity scheme. As an alternative scheme, transmit antenna selection (TAS) has been proposed by [3][5], when multiple antennas are deployed at the transmitter. Considering feedback overhead and signal processing cost, a single transmit antenna is selected in TAS to maximize the signal-to-noise ratio (SNR) at the secondary user receiver (SRX). In addition, a general maximal ratio combining (MRC) is applied among several receiver antennas to enhance the performance of the secondary users' network.

In contrast to MRT and TAS, the distributed cyclic delay diversity (dCDD) has been proposed as a practical transmit diversity scheme for cyclic-prefixed single carrier (CP-SC)

K. J. Kim is with Mitsubishi Electric Research Laboratories (MERL), Cambridge, MA, USA.

H. Liu is with Shandong Jiaotong University, Jinan, China, and Inha University, Korea.

M. D. Renzo is with the Laboratoire des Signaux et Systèmes, CNRS, CentraleSupélec, Univ Paris Sud, Université Paris-Saclay, 3 rue Joliot Curie, Plateau du Moulon, 91192, Gif-sur-Yvette, France. (e-mail: marco.direnzo@12s.centralesupelec.fr).

H. V. Poor is with the Department of Electrical Engineering, Princeton University, Princeton, NJ, USA.

This work was supported in part by the U.S. National Science Foundation under Grants CNS-1702808 and ECCS-1647198. systems [6]. Without explicit channel feedback from the receiver side, dCDD improves the reliability of a message by transmitting the same message over multiple channels having different channel characteristics. Several works [7]-[10] have applied the conventional CDD for several applications. In contrast to the conventional CDD, which applies a cyclic delay among antennas deployed at the same transmitter, dCDD applies a cyclic delay among antennas, each of which is deployed at a different transmitter. By proper design of the permutation matrix that circularly shifts the symbol block, intersymbol interference (ISI)-free channel matrix can be generated. The size of the symbol block and the maximum number of multipath components of the channel that transmits this symbol block determine the number of transmitters for dCDD. It has been verified by [6] that the full diversity gain with a higher coding gain can be achieved over those of [3]-[5], [11]-[13] without exact knowledge of CSI at the transmitter side. This performance gain advantage will be beneficial of secondary users in reusing the radio spectrum licensed to primary users.

There are several existing works [12], [13] for the spectrum sharing systems that use $\mathrm{CP}-\mathrm{SC}$ transmissions. In contrast to these existing works, we can summarize our main contributions as follows.

- To achieve the transmit diversity gain, $\mathrm{dCDD}$ is employed between the control unit (CU) and a finite number of secondary user remote radio heads (SU-RRHs). We use a mathematical analysis fit to finite-sized cooperative spectrul sharing systems. For a secondary users' network, we investigate the impact of the isotropic random location of the primary user transmitter (PTX) on the outage probability. Thus, the use of $\mathrm{dCDD}$ in the spectrum sharing system is one of the key distinctions from [12], [13].

- Due to the random location of the PTX within the secondary users' network, a more practical channel model, which is somewhat similar to that of [14]-[17], is used. The co-existence of line-of-sight ( $\mathrm{LoS})$ and non-line-ofsight (nLoS) paths is modeled by using a time-sharing factor [18], which is being distributed by the bernoulli process.

- We provide an analytical framework jointly taking into account a different degree of RRH cooperation via dCDD, frequency selectivity fading, isotropic random location of the PTX over over the co-existing LoS and nLoS paths. For this new setting for the spectrum sharing system, a new expression for the spatially averaged signal-to- 
interference ratio (SA-SIR) is derived. SA-SINR is derived.

- We analyze the achievable diversity gain from the outage probability.

\section{A. Notation}

$\mathbb{C}$ denotes the set of complex numbers; $\mathcal{C N}\left(\mu, \sigma^{2}\right)$ denotes the circularly symmetric complex Gaussian distribution with the mean $\mu$ and the variance $\sigma^{2} ; F_{\varphi}(\cdot)$ and $f_{\varphi}(\cdot)$, respectively, denote the cumulative distribution function (CDF) and probability density function (PDF) of the random variable (RV) $\varphi$; $E\{\cdot\}$ denotes expectation. A length of a vector $\boldsymbol{a}$ is denoted by $\mathbb{L}(\boldsymbol{a})$.

\section{System AND ChanNel Model}

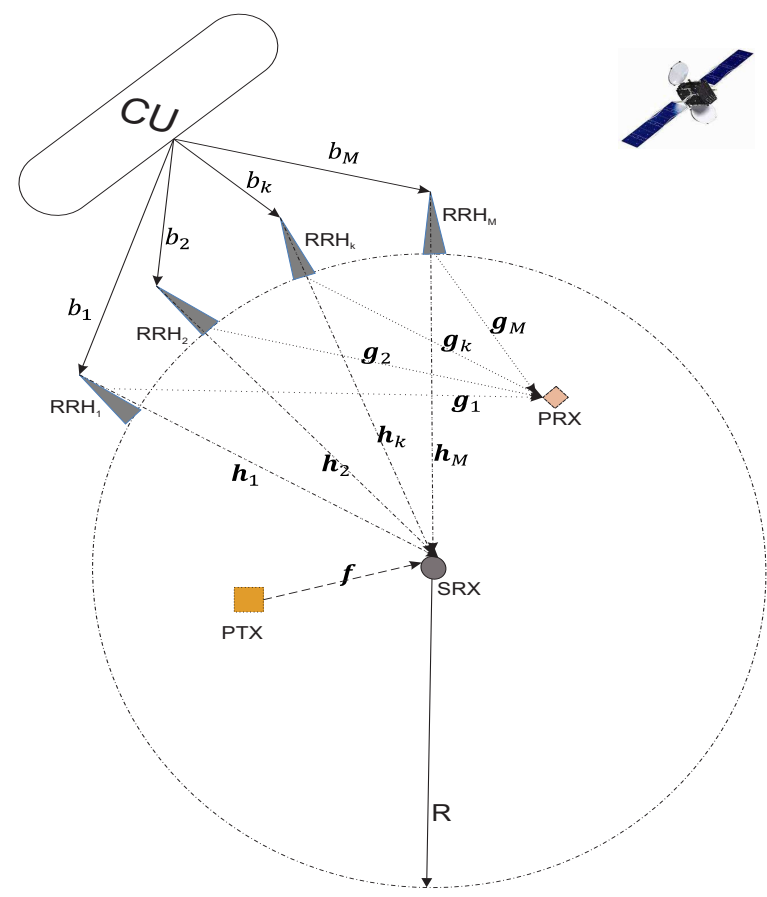

Fig. 1. Block diagram of the considered dCDD-based spectrum sharing system with the PTX placed at random within the secondary user's cell.

Fig. 1 illustrates a block diagram of the considered dCDDbased spectrum sharing system comprising one PTX and primary user receiver (PRX) in the primary licensed frequency band. A circular shaped communication cell of radius $R$ for the secondary users is formed around the SRX. In the cell, the location of the PTX is assumed to be isotropic, whereas the PRX is placed at a fixed location. A group of secondary SU-RRHs are distributed over the circumference of the cell. A secondary user network consisting of the CU, $M$ SU-RRHs, and SRX shares the primary users' licensed frequency band subject to interference constraints imposed by the PRX. The CU controls $M$ single antenna equipped RRHs, $\left\{\mathrm{RRH}_{m}\right\}_{m=1}^{M}$, via dedicated highly reliable backhauls, $\left\{b_{m}\right\}_{m=1}^{M}$. The CU forms an information data being transmitted by SU-RRHs simultaneously to the SRX by using dCDD , so that each SU-RRH requires only a simple hardware and transmission power in communicating with the SRX. A receiving unit for Global Navigation Satellite System (GNSS) signal is installed at the front-end of each node, so that SU-RRHs can receive and transmit the same information data at the same time when they are operating for dCDD. Due to the use of half-duplex transceivers in every nodes, they are allowed to either send or receive data at a time.

Since the number of SU-RRHs for dCDD is limited by the symbol block size and the maximum multipath path components over the frequency fading channel in the secondary users' network [6], this paper investigates only a finite-sized cooperative spectrum sharing system comprising a finite number of SU-RRHs for dCDD and a single SRX. By employing appropriate channel sounding scheme or channel reciprocity [19], [20], we further assume that the SRX is able to know the maximum number of multipath components over the channels from SU-RRHs to itself. The following channels are assumed in the considered system.

- Channels from SU-RRHs to the SRX: A multipath channel, $\boldsymbol{h}_{m}$, from the $m$ th SU-RRH to the SRX is given by

$$
\boldsymbol{h}_{m}=\sqrt{R^{-\epsilon_{\mathrm{L}}}} \tilde{\boldsymbol{h}}_{m}
$$

where $\tilde{\boldsymbol{h}}_{m}$ denotes a frequency selective fading channel with $N_{h} \triangleq\left\{\mathbb{L}\left(\tilde{\boldsymbol{h}}_{m}\right)\right\}_{m=1}^{M}$ multipath components, and $\epsilon_{\mathrm{L}}$ denoting the path loss exponent of all the line-of-sight (LoS) paths over all $\left\{\tilde{\boldsymbol{h}}_{m}\right\}_{m=1}^{M}$.

- A channel from the PTX to the SRX: A multipath channel from the PTX to the SRX is given by

$$
\boldsymbol{f}=\mathbb{I}_{\mathrm{L}} \sqrt{\left(d_{2}\right)^{-\epsilon_{\mathrm{L}}}} \tilde{\boldsymbol{f}}_{\mathrm{L}}+\left(1-\mathbb{I}_{\mathrm{L}}\right) \sqrt{\left(d_{2}\right)^{-\epsilon_{\mathrm{nL}}}} \tilde{\boldsymbol{f}}_{\mathrm{nL}}
$$

where $\tilde{f}_{\mathrm{L}}$ and $\tilde{f}_{\mathrm{nL}}$ identify frequency selective fading channels over LoS and non-line-of-sight (nLoS) paths with $N_{\mathrm{L}} \triangleq \mathbb{L}\left(\tilde{f}_{\mathrm{L}}\right)$ and $N_{\mathrm{nL}} \triangleq \mathbb{L}\left(\tilde{f}_{\mathrm{nL}}\right)$ multipath components. The path loss exponent over $\tilde{f}_{\mathrm{nL}}$ is denoted by $\epsilon_{\mathrm{nL}}$. The indicator function, $\mathbb{I}_{\mathrm{L}}$, is used to model the random selection of LoS and $\mathrm{nLoS}$ path with probability $P_{r}\left(\mathbb{I}_{\mathrm{L}}\right)=\mathcal{F}$ and $P_{r}\left(\mathbb{I}_{\mathrm{nL}}\right)=1-\mathcal{F}$ with time-sharing factor $\mathcal{F}$ [18]. Owing to the random location of the PTX within the secondary users' network, we model it using a bernoulli process. Distance $d_{2}$ from the PTX to the SRX is distributed as follows:

$$
f_{d_{2}}\left(y_{2}\right)=2 y_{2} / R^{2} \text { for } 0 \leq y_{2} \leq R .
$$

- Channels from SU-RRHS to the PRX : When $M$ SURRHs are selected for dCDD, they influence the PRX, so that a channel from the $m$ th SU-RRH to the PRX is given by

$$
\boldsymbol{g}_{m}=\sqrt{\left(d_{3, m}\right)^{-\epsilon_{\mathrm{L}}}} \tilde{\boldsymbol{g}}_{m}
$$

where $\tilde{\boldsymbol{g}}_{m}$ identifies the frequency selective fading channel with $N_{g} \triangleq\left\{\mathbb{L}\left(\tilde{\boldsymbol{g}}_{m}\right)\right\}_{m=1}^{M}$. A definite distance from the $m$ th RRH to the PRX is given by $d_{3, m}$.

- All frequency selective fading channels are assumed to be independent and identically distributed (i.i.d.). We also assume that all channels are constant over one 
data transmission interval due to a quasi-static channel assumption, but different from and independent of those for other transmission intervals.

\section{A. dCDD for CP-SC Transmissions}

As a transmission scheme, CP-SC transmissions [11]-[13] are used for the primary and secondary user networks. To remove ISI being caused by a multipath channel between two nodes, the last $N_{p}$ modulation symbols from a transmission symbol block $s \in \mathbb{C}^{B \times 1}$ are appended to the front of $s[6]$. The size of symbol block $s$ is denoted by $B$. We assume that $E\{\boldsymbol{s}\}=\mathbf{0}$ and $E\left\{\|\boldsymbol{s}\|^{2}\right\}=\boldsymbol{I}_{B}$. To make an ISI-free right circulant channel matrix, the CP length, $N_{p}$, and the CDD delay, for example, $\Delta_{m}$ for the $m$ th SU-RRH, play key roles in generating an equivalent ISI-free right circulant channel matrix by satisfying the following two conditions [6]:

$$
N_{p} \geq N_{h} \text { and } \Delta_{m}=(m-1) N_{p} .
$$

Since CP-SC transmissions use a block transmission, the maximum allowable number of SU-RRHs for dCDD is determined by $K=1+\left\lfloor B / N_{p}\right\rfloor$, where $\lfloor\cdot\rfloor$ denotes the floor function. However, in this paper, we assume that there are only $M \leq K$ SU-RRHs in the secondary user network.

Due to dCDD, the received signal at the SRX, after removing the $\mathrm{CP}$ signal, is given by

$$
\begin{aligned}
\boldsymbol{r}= & \sqrt{P_{s}} \sum_{m=1}^{M} R^{-\epsilon_{\mathrm{L}} / 2} \tilde{\boldsymbol{H}}_{m} \tilde{\boldsymbol{s}}_{m}+ \\
& \sqrt{P_{P}}\left(\mathbb{I}_{\mathrm{L}} d_{2}^{-\epsilon_{\mathrm{L}} / 2} \tilde{\boldsymbol{F}}_{\mathrm{L}}+\left(1-\mathbb{I}_{\mathrm{nL}}\right) d_{2}^{-\epsilon_{\mathrm{L}} / 2} \tilde{\boldsymbol{F}}_{\mathrm{nL}}\right) \tilde{\boldsymbol{x}}_{p}+\boldsymbol{z}_{R}
\end{aligned}
$$

where $\tilde{\boldsymbol{s}}_{m} \triangleq \boldsymbol{P}_{B}^{\Delta_{m}} \boldsymbol{s}_{m}, P_{P}$ is the transmission power at the PTX. Permutation shifting matrix $\boldsymbol{P}_{B}^{\Delta_{m}}$ is obtained from the identity matrix $\boldsymbol{I}_{B}$ by circularly shifting down by $\Delta_{m}$. The transmission power at the SU-RRHs is $P_{s}$, which is given by the following constraints [13]:

$$
P_{s}=\min \left(P_{T}, \frac{I_{p}}{\max _{m=1, \ldots, M} P_{G} d_{3, m}^{-\epsilon_{\mathrm{L}}}\left\|\tilde{\boldsymbol{g}}_{m}\right\|^{2}}\right)
$$

where $P_{T}$ and $I_{p}$ respectively denotes the maximum allowable transmission power at the SU-RRHs and the peak allowable interference at the PRX. The transmission power at the PRX is denoted by $P_{G}$. Channel matrices, $\tilde{\boldsymbol{H}}_{m}, \tilde{\boldsymbol{F}}_{\mathrm{L}}$, and $\tilde{\boldsymbol{F}}_{\mathrm{nL}}$ are right circulant respectively specified by $\tilde{\boldsymbol{h}}_{m}, \tilde{\boldsymbol{f}}_{\mathrm{L}}$, and $\tilde{\boldsymbol{f}}_{\mathrm{nL}}$. Since $\max \left\{N_{h}, N_{\mathrm{L}}, N_{\mathrm{nL}}, N_{g}\right\}$ is smaller than block size $B$, a zero padding is required in the representation of right circulant channel matrices. The transmission symbol block from the PTX is given by $\boldsymbol{x}_{p}$ with $E\left\{\boldsymbol{x}_{p}\right\}=\mathbf{0}$ and $E\left\{\boldsymbol{x}_{p} \boldsymbol{x}_{p}^{H}\right\}=\boldsymbol{I}_{B}$, and $E\left\{\tilde{\boldsymbol{s}}_{m} \boldsymbol{x}_{p}^{H}\right\}=\mathbf{0}$. The additive noise over the frequency selective fading channels is denoted by $\boldsymbol{z}_{R} \sim \mathcal{C N}\left(\mathbf{0}, \sigma_{z}^{2} \boldsymbol{I}_{B}\right)$.

\section{Performance Analysis in Interference Limited REGION}

From (6), the SIR measured at the SRX is given by

$$
\gamma_{\mathrm{SIR}} \triangleq \frac{P_{s} \sum_{m=1}^{M} R^{-\epsilon_{\mathrm{L}}}\left\|\tilde{\boldsymbol{h}}_{m}\right\|^{2}}{P_{P}\left(\mathcal{F} d_{2}^{-\epsilon_{\mathrm{L}}}\left\|\tilde{\boldsymbol{f}}_{\mathrm{L}}\right\|^{2}+(1-\mathcal{F}) d_{2}^{-\epsilon_{\mathrm{nL}}}\left\|\tilde{\boldsymbol{f}}_{\mathrm{nL}}\right\|^{2}\right)}
$$

$$
=\min \left(P_{T}, I_{p} / X\right) Y
$$

where $\quad X \triangleq \max _{m=1, \ldots, M} P_{G} d_{3, m}^{-\epsilon_{\mathrm{L}}}\left\|\tilde{\boldsymbol{g}}_{m}\right\|^{2}, \quad Y \triangleq \frac{A}{B} \quad$ with $A \triangleq \sum_{m=1}^{M} R^{-\epsilon_{\mathrm{L}}}\left\|\tilde{\boldsymbol{h}}_{m}\right\|^{2}$ and $B \triangleq P_{P}\left(\mathcal{F} d_{2}^{-\epsilon_{\mathrm{L}}}\left\|\tilde{\boldsymbol{f}}_{\mathrm{L}}\right\|^{2}+\right.$ $\left.(1-\mathcal{F}) d_{2}^{-\epsilon_{\mathrm{nL}}}\left\|\tilde{f}_{\mathrm{nL}}\right\|^{2}\right)$. Note that when we use maximum likelihood type detector, for example, QRD-M detector [21], we can obtain (8).

\section{A. Distributions of the SA-SIR}

Owing to the random location of the PTX within the secondary users' cell, we need to compute the SA-SIR. Extending from the analysis of [13], the CDF of the SA-SIR is given by

$$
\begin{aligned}
F_{\gamma}(x)= & \underbrace{E\left\{F_{Y}\left(x / P_{T} \mid d_{2}\right)\right\}}_{J_{1}} F_{X}(\mu)+ \\
& \underbrace{E\left\{\int_{\mu}^{\infty} F_{Y}\left(x t / I_{p} \mid d_{2}\right)\right\} f_{X}(t)}_{J_{2}} d t
\end{aligned}
$$

where $\mu \triangleq \frac{I_{p}}{P_{T}}$. To compute (9), we can readily find the following distributions and density of RV $X$ :

$$
\begin{aligned}
F_{X}(x) & =\prod_{m=1}^{M}\left(1-\frac{\Gamma_{u}\left(N_{g}, x / P_{G} d_{3, m}^{-\epsilon_{\mathrm{L}}}\right)}{\Gamma\left(N_{g}\right)}\right) \\
& =1+\Upsilon e^{-\tilde{\beta} x} x^{\tilde{l}}, \\
f_{X}(x) & =\Upsilon\left[\tilde{l} e^{-\tilde{\beta} x} x^{\tilde{l}-1}-\tilde{\beta} e^{-\tilde{\beta} x} x^{\tilde{l}}\right]
\end{aligned}
$$

where $\Gamma(\cdot)$ and $\Gamma_{u}(\cdot)$ respectively denote the complete gamma function and upper incomplete gamma function. In addition,

$$
\begin{aligned}
\tilde{\beta} \triangleq & \sum_{t=1}^{m} \frac{1}{P_{G} d_{3, q_{t}}^{-\epsilon_{\mathrm{L}}}}, \tilde{l} \triangleq \sum_{t=1}^{m} \ell_{t}, \text { and } \\
\Upsilon \triangleq & \sum_{m=1}^{M}(-1)^{m} \sum_{q_{1}=1}^{M-m+1} \cdots \sum_{q_{m}=q_{m-1}+1}^{M} \\
& \sum_{\ell_{1}=0}^{N_{g}-1} \cdots \sum_{\ell_{m}=0}^{N_{g}-1} \prod_{t=1}^{m}\left(\frac{1}{\ell_{t} !\left(P_{G} d_{3, q_{t}}^{-\epsilon_{\mathrm{L}}}\right)_{t}}\right) .
\end{aligned}
$$

According to the analysis provided in [6], the distribution of $\mathrm{RV} A$, is given by

$$
F_{A}(x)=1-\frac{\Gamma_{u}\left(M N_{h}, x / P_{s} R^{-\epsilon_{\mathrm{L}}}\right)}{\Gamma\left(M N_{h}\right)} .
$$

Furthermore, from CP-SC transmissions, the conditional density of primary user's interference power received at the SRX at a given distance $d_{2}$, denoted by $\mathrm{RV} B$, is given by

$$
\begin{aligned}
f_{B}\left(x \mid d_{2}\right)= & \frac{\mathcal{F}}{\Gamma\left(N_{\mathrm{L}}\right)\left(P_{P} d_{2}^{-\epsilon_{\mathrm{L}}}\right)^{N_{\mathrm{L}}}} x^{N_{\mathrm{L}}-1} e^{-\frac{x}{P_{P} d_{2}^{-\epsilon_{\mathrm{L}}}}}+ \\
& \frac{(1-\mathcal{F})}{\Gamma\left(N_{\mathrm{nL}}\right)\left(P_{P} d_{2}^{-\epsilon_{\mathrm{nL}}}\right)^{N_{\mathrm{nL}}}} x^{N_{\mathrm{nL}}-1} e^{-\frac{x}{P_{P} d_{2}^{-\epsilon_{\mathrm{nL}}}}(14)}
\end{aligned}
$$

with the density of $d_{2}$ given by $f_{d_{2}}\left(y_{2}\right)=\frac{2 y_{2}}{R^{2}}, 0 \leq y_{2} \leq R$.

After having some manipulations, the expression for $J_{1}$ in (9) can be evaluated as (15) at the next page. In (15), we have defined $\rho \triangleq \frac{P_{P}}{P_{s}}$. In addition, $G_{p, q}^{m, n}(\cdot \mid \cdot, \cdot \cdot)$ denotes the Meijer-G functions [22, sec. (2.24)]. Due to space limitation, we skip the derivation of (15). 


$$
\begin{aligned}
J_{1}= & 1-\frac{2 \rho^{-N_{\mathrm{L}}}}{\epsilon_{\mathrm{L}} \Gamma\left(N_{\mathrm{L}}\right)}\left(\frac{x}{P_{T}}\right)^{-N_{\mathrm{L}}} \sum_{m=0}^{M N_{h}-1} \frac{1}{m !} G_{2,2}^{2,1}\left(\frac{x \rho}{P_{T}} \mid \begin{array}{c}
1, N_{\mathrm{L}}+2 / \epsilon_{\mathrm{L}}+1 \\
N_{\mathrm{L}}+2 / \epsilon_{\mathrm{L}}, N_{\mathrm{L}}+m
\end{array}\right)- \\
& \frac{2 \rho^{-N_{\mathrm{nL}}} R^{N_{\mathrm{nL}}\left(\epsilon_{\mathrm{nL}}-\epsilon_{\mathrm{L}}\right)}}{\epsilon_{\mathrm{nL}} \Gamma\left(N_{\mathrm{nL}}\right)}\left(\frac{x}{P_{T}}\right)^{-N_{\mathrm{nL}}} \sum_{m=0}^{M N_{h}-1} \frac{1}{m !} G_{2,2}^{2,1}\left(\frac{x \rho R^{\epsilon_{\mathrm{L}}-\epsilon_{\mathrm{nL}}}}{P_{T}} \mid \begin{array}{c}
1, N_{\mathrm{nL}}+2 / \epsilon_{\mathrm{nL}}+1 \\
N_{\mathrm{nL}}+2 / \epsilon_{\mathrm{nL}}, N_{\mathrm{nL}}+m
\end{array}\right) .
\end{aligned}
$$

A more challenging task is the computation of $J_{2}$ in (9). Since the integral solution of $J_{2}$ in (9) does not exist, we apply an approximation in the asymptotic region provided in the following theorem.

Theorem 1: The approximate expression for $J_{2}$ in the asymptotic region is given by (16).

Proof: According to [23], we first approximate the following Meijer-G function as

$$
\begin{aligned}
& G_{2,2}^{2,1}\left(x \mid \begin{array}{c}
1, N_{\mathrm{L}}+2 / \epsilon_{\mathrm{L}}+1 \\
N_{\mathrm{L}}+2 / \epsilon_{\mathrm{L}}, N_{\mathrm{L}}+m
\end{array}\right)^{x \rightarrow 0} \underset{x^{N_{\mathrm{L}}+2 / \epsilon_{\mathrm{L}}}}{\approx} \approx\left(m-2 / \epsilon_{\mathrm{L}}\right) \Gamma\left(N_{\mathrm{L}}+2 / \epsilon_{\mathrm{L}}\right)+x^{N_{\mathrm{L}}+m} \Gamma\left(m+N_{\mathrm{L}}\right) \\
& \Gamma\left(2 / \epsilon_{\mathrm{L}}+1-m\right) .
\end{aligned}
$$

And then after conducting some manipulations, we can derive (16). Note that since we can readily extract expressions for $C_{1}, C_{2}, D_{1}$, and $D_{2}$, we do not provide their corresponding expressions.

\section{B. Outage Probability}

Using (15) and (16), the approximate outage probability at a given SIR threshold $O_{\text {th }}$, is given by (18).

\section{Asymptotic Diversity Gain Analysis}

Using (17), we can approximate $J_{1}$ as in (19), where we have used the approach provided in [23] for an asymptotic approximation of $(a)$. Similarly, $C_{1}, C_{2}, D_{1}$ and $D_{2}$ are approximately proportional to $\left(x / I_{p}\right)^{N_{h} M}$. Thus, as $P_{T} \rightarrow \infty$ and $I_{p} \rightarrow \infty$, while maintaining $\mu$ a constant, the maximum diversity gain, $G_{d}=M N_{h}$, can be achievable by the use of dCDD in the secondary users' network. Thus, we can see that a different time sharing on LoS and nLoS paths, and primary users' system and channel parameters have no effect on the diversity gain.

\section{Simulations}

The following simulation setup is considered:

- $B=192$ and $N_{p}=64$, so that $K=3$ is the maximum number of RRH for dCDD.

- Quadrature phase-shift keying (QPSK) modulation is used.

- Different path-loss exponents are assumed to be $\epsilon_{1}=$ $2.09, \epsilon_{2}=3.75$, and $\epsilon_{3}=3.75$ in the considered system [17].

- SRX is placed at the center of a circular shaped secondary users' communication cell of radius $R$, within which the PRX is placed at $\left(\Delta_{x}, \Delta_{y}\right)$.

- Three SU-RRHs are placed at $R^{j \pi / 2}, R^{j(\pi / 2-\pi / 10)}$, and $R^{j(\pi / 2+\pi / 10)}$.
The curves obtained via link-level simulations are denoted by Ex. Analytical performance curves are denoted by An. The SIR threshold causing an outage is fixed at $O_{\mathrm{th}}=1 \mathrm{~dB}$.

\section{A. Outage Probability Analysis}

Fig. 2 shows the outage probability for various values of $N_{h}$ at fixed values of $\left(M=2, N_{\mathrm{L}}=2, N_{\mathrm{nL}}=3, N_{g}=\right.$ $2, R=10, \mathcal{F}=0.6, P_{P}=3 \mathrm{~dB}, P_{s}=1 \mathrm{~dB}, P_{g}=$ $\left.1 \mathrm{~dB}, \Delta_{x}=1, \Delta_{y}=3\right)$. This figure shows the accuracy of our derivations for the outage probability. Thus, in the sequel, we will mainly use the analytically derived outage probability for the performance analysis w.l.o.g..

Fig. 3 compares the outage probability of the proposed system over the existing work (denoted by conv. in the curves), which does not use dCDD. Only one SU-RRH, which has the largest SIR among them, is selected [3]-[5], [12], [13]. This figure shows that dCDD results in a better outage probability even with a small number of SU-RRHs in the secondary users' network. As more RRHs are available for dCDD, the performance gap will be increased. If we measure the slopes of the curves in this figure, they are identical when $M N_{h}$ is same. This will be further investigated in Fig. 4.

In Fig. 4, we investigate different values for $N_{h}, \mathcal{F}, R$, and $\left(N_{\mathrm{L}}, N_{\mathrm{nL}}\right)$. In the considered all cases, dCDD provides a better outage probability over the conventional one. This figure also shows that

- As $N_{h}$ increases, a better outage probability is obtained due to a higher multipath diversity gain.

- A different degree of co-existence of LoS and nLoS paths, which is specified by $\mathcal{F}$, does not influence on the slope of the outage probability curve in the asymptotic region of $P_{T}$. Since we assume a constant $\mu$, this corresponds to an asymptotic region of $I_{p}$ as well.

- A larger radius of the secondary users' communication cell, which is denoted by $2 R$, affects adversely the outage probability due to a greater distance between SU-RRHs and the SRX.

- A different number of multipath components over the channel from the PTX to the SRX, does not change the slope of the outage probability curve in the asymptotic region, for example, $\left(N_{\mathrm{L}}=2, N_{\mathrm{nL}}=3\right)$ vs. $\left(N_{\mathrm{L}}=\right.$ $\left.5, N_{\mathrm{nL}}=6\right)$ when $M N_{h}$ is same.

\section{CONCLUSions}

In this paper, we have investigated the performance enhancement of the secondary users for cooperative spectrum sharing systems. As the transmit diversity scheme, dCDD has been employed between the CU and distributed SU-RRHs. From the performance analysis, it has been seen that dCDD 


$$
\begin{aligned}
J_{2}= & 1-F_{X}(\mu)-\frac{2 \mathcal{F} \Upsilon \tilde{l}}{\epsilon_{\mathrm{L}} \Gamma\left(N_{\mathrm{L}}\right)} \sum_{m=0}^{M N_{h}-1} \frac{1}{m !}\left(\left(\frac{\rho x}{I_{p}}\right)^{2 / \epsilon_{\mathrm{L}}} \Gamma\left(m-2 / \epsilon_{\mathrm{L}}\right) \Gamma\left(N_{\mathrm{L}}+2 / \epsilon_{\mathrm{L}}\right)(\tilde{\beta})^{-\tilde{l}-2 / \epsilon_{\mathrm{L}}} \Gamma_{u}\left(\tilde{l}+2 / \epsilon_{\mathrm{L}}, \tilde{\beta} \mu\right)+\right. \\
& \left.\left(\frac{\rho x}{I_{p}}\right)^{m} \Gamma\left(2 / \epsilon_{\mathrm{L}}+1-m\right) \Gamma\left(N_{\mathrm{L}}+m\right)(\tilde{\beta})^{-\tilde{l}-m} \Gamma_{u}(\tilde{l}+m, \tilde{\beta} \mu)\right)+ \\
& \frac{2 \mathcal{F} \Upsilon \tilde{\beta}}{\epsilon_{\mathrm{L}} \Gamma\left(N_{\mathrm{L}}\right)} \sum_{m=0}^{M N_{h}-1} \frac{1}{m !}\left(\left(\frac{\rho x}{I_{p}}\right)^{2 / \epsilon_{\mathrm{L}}} \Gamma\left(m-2 / \epsilon_{\mathrm{L}}\right) \Gamma\left(N_{\mathrm{L}}+2 / \epsilon_{\mathrm{L}}\right)(\tilde{\beta})^{-\tilde{l}-2 / \epsilon_{\mathrm{L}}-1} \Gamma_{u}\left(\tilde{l}+2 / \epsilon_{\mathrm{L}}+1, \tilde{\beta} \mu\right)+\right. \\
& \left.\left(\frac{\rho x}{I_{p}}\right)^{m} \Gamma\left(2 / \epsilon_{\mathrm{L}}+1-m\right) \Gamma\left(N_{\mathrm{L}}+m\right)(\tilde{\beta})^{-\tilde{l}-m-1} \Gamma_{u}(\tilde{l}+m+1, \tilde{\beta} \mu)\right)- \\
& \frac{2(1-\mathcal{F}) \Upsilon \tilde{l}}{\epsilon_{\mathrm{L}} \Gamma\left(N_{\mathrm{L}}\right)} \sum_{m=0}^{M N_{h}-1} \frac{1}{m !}\left(\left(\frac{\rho x R_{\mathrm{L}}-\epsilon_{\mathrm{nL}}}{I_{p}}\right)^{2 / \epsilon_{\mathrm{L}}} \Gamma\left(m-2 / \epsilon_{\mathrm{L}}\right) \Gamma\left(N_{\mathrm{L}}+2 / \epsilon_{\mathrm{L}}\right)(\tilde{\beta})^{-\tilde{l}-2 / \epsilon_{\mathrm{L}}} \Gamma_{u}\left(\tilde{l}+2 / \epsilon_{\mathrm{L}}, \tilde{\beta} \mu\right)+\right. \\
& \left.\left(\frac{\rho x R^{\epsilon_{\mathrm{L}}-\epsilon_{\mathrm{nL}}}}{I_{p}}\right)^{m} \Gamma\left(2 / \epsilon_{\mathrm{L}}+1-m\right) \Gamma\left(N_{\mathrm{L}}+m\right)(\tilde{\beta})^{-\tilde{l}-m} \Gamma_{u}(\tilde{l}+m, \tilde{\beta} \mu)\right)+ \\
& \frac{2(1-\mathcal{F}) \Upsilon \tilde{\beta}}{\epsilon_{\mathrm{L}} \Gamma\left(N_{\mathrm{L}}\right)} \sum_{m=0}^{M N_{h}-1} \frac{1}{m !}\left(\left(\frac{\rho x R^{\epsilon_{\mathrm{L}}-\epsilon_{\mathrm{n}}}}{I_{p}}\right)^{2 / \epsilon_{\mathrm{L}}} \Gamma\left(m-2 / \epsilon_{\mathrm{L}}\right) \Gamma\left(N_{\mathrm{L}}+2 / \epsilon_{\mathrm{L}}\right)(\tilde{\beta})^{-\tilde{l}-2 / \epsilon_{\mathrm{L}}-1} \Gamma_{u}\left(\tilde{l}+2 / \epsilon_{\mathrm{L}}+1, \tilde{\beta} \mu\right)+\right. \\
& \left.\left(\frac{\rho x R^{\epsilon_{\mathrm{L}}-\epsilon_{\mathrm{nL}}}}{I_{p}}\right)^{m} \Gamma\left(2 / \epsilon_{\mathrm{L}}+1-m\right) \Gamma\left(N_{\mathrm{L}}+m\right)(\tilde{\beta})^{-\tilde{l}-m-1} \Gamma_{u}(\tilde{l}+m+1, \tilde{\beta} \mu)\right) \\
\triangleq & 1-F_{X}(\mu)-C_{1}+C_{2}-D_{1}+D_{2} .
\end{aligned}
$$

$$
\begin{aligned}
P_{\text {outage }}\left(O_{\text {th }}\right)= & 1-F_{X}(\mu) \frac{2 \rho^{-N_{\mathrm{L}}}}{\epsilon_{\mathrm{L}} \Gamma\left(N_{\mathrm{L}}\right)}\left(\frac{O_{\mathrm{th}}}{P_{T}}\right)^{-N_{\mathrm{L}}} \sum_{m=0}^{M N_{h}-1} \frac{1}{m !} G_{2,2}^{2,1}\left(\frac{O_{\mathrm{th}} \rho}{P_{T}} \mid \begin{array}{c}
1, N_{\mathrm{L}}+2 / \epsilon_{\mathrm{L}}+1 \\
N_{\mathrm{L}}+2 / \epsilon_{\mathrm{L}}, N_{\mathrm{L}}+m
\end{array}\right)- \\
& F_{X}(\mu) \frac{2 \rho^{-N_{\mathrm{nL}}} R^{N_{\mathrm{nL}}\left(\epsilon_{\mathrm{nL}}-\epsilon_{\mathrm{L}}\right)}}{\epsilon_{\mathrm{nL}} \Gamma\left(N_{\mathrm{nL}}\right)}\left(\frac{O_{\mathrm{th}}}{P_{T}}\right)^{-N_{\mathrm{nL}}} \sum_{m=0}^{M N_{h}-1} \frac{1}{m !} G_{2,2}^{2,1}\left(\frac{O_{\mathrm{th}} \rho R^{\epsilon_{\mathrm{L}}-\epsilon_{\mathrm{nL}}}}{P_{T}} \mid \begin{array}{c}
1, N_{\mathrm{nL}}+2 / \epsilon_{\mathrm{nL}}+1 \\
N_{\mathrm{nL}}+2 / \epsilon_{\mathrm{nL}}, N_{\mathrm{nL}}+m
\end{array}\right) \\
& -\left.C_{1}\right|_{x=O_{\text {th }}}+\left.C_{2}\right|_{x=O_{\text {th }}}-\left.D_{1}\right|_{x=O_{\text {th }}}+\left.D_{2}\right|_{x=O_{\text {th }}} .
\end{aligned}
$$

$$
\begin{aligned}
& J_{1} P_{T} \rightarrow \infty 1-\frac{2}{\epsilon_{\mathrm{L}} \Gamma\left(N_{\mathrm{L}}\right)} \sum_{m=0}^{M N_{h}-1}\left(\frac{\rho^{2 / \epsilon_{\mathrm{L}}}}{m !}\left(\frac{x}{P_{T}}\right)^{2 / \epsilon_{\mathrm{L}}} \Gamma\left(m-2 / \epsilon_{\mathrm{L}}\right) \Gamma\left(N_{\mathrm{L}}+2 / \epsilon_{\mathrm{L}}\right)+\frac{\rho^{m}}{m !}\left(\frac{x}{P_{T}}\right)^{m} \Gamma\left(m+N_{\mathrm{nL}}\right)\right. \\
& \left.\Gamma\left(2 / \epsilon_{\mathrm{L}}+1-m\right)\right)-\frac{2}{\epsilon_{\mathrm{nL}} \Gamma\left(N_{\mathrm{nL}}\right)} \sum_{m=0}^{M N_{h}-1}\left(\frac{\left(\rho R^{\epsilon_{\mathrm{L}}-\epsilon_{\mathrm{nL}}}\right)^{2 / \epsilon_{\mathrm{nL}}}}{m !}\left(\frac{x}{P_{T}}\right)^{2 / \epsilon_{\mathrm{nL}}} \Gamma\left(m-2 / \epsilon_{\mathrm{nL}}\right)\right. \\
& \left.\Gamma\left(N_{\mathrm{nL}}+2 / \epsilon_{\mathrm{nL}}\right)+\frac{\left(\rho R^{\epsilon_{\mathrm{L}}-\epsilon_{\mathrm{nL}}}\right)^{m}}{m !}\left(\frac{x}{P_{T}}\right)^{m} \Gamma\left(m+N_{\mathrm{nL}}\right) \Gamma\left(2 / \epsilon_{\mathrm{nL}}+1-m\right)\right) \\
& \stackrel{(a)}{\propto}\left(\frac{x}{P_{T}}\right)^{N_{h} M}
\end{aligned}
$$

provides a better outage probability over the existing work, which uses only one RRH as the secondary user transmitter. Furthermore, it has been justified from the simulations that the maximum diversity gain can be achieved. It has been seen that the primary users' system and channel parameters have no impacts on the achievable diversity gain of the secondary users' network.

\section{REFERENCES}

[1] T. K. Y. Lo, "Maximum ratio transmission," IEEE Trans. Commun., vol. 47, no. 10, pp. 1458-1461, Oct. 1999.

[2] K. J. Kim, T. Khan, and P. Orlik, "Performance analysis of cooperative systems with unreliable backhauls and selection combining," IEEE Trans. Veh. Technol., vol. 66, no. 3, pp. 2448-2461, Mar. 2017.
[3] V. M. Blagojevic and P. N. Ivanis, "Ergodic capacity for TAS/MRC spectrum sharing cognitive radio," IEEE Commun. Lett., vol. 16, no. 3 , pp. 312-323, Mar. 2012.

[4] P. L. Yeoh, M. Elkashlan, T. Q. Trung, N. Yang, and D. B. da Costa, "Transmit antenna selection for interference management in cognitive relay networks," IEEE Trans. Veh. Technol., vol. 63, no. 7, pp. 32503262, 2014.

[5] M. Hanif, H. C. Yang, and M. S. Alouini, "Transmit antenna selection for power adaptive underlay cognitive radio with instantaneous interference constraint," IEEE Trans. Commun., vol. 65, no. 6, pp. 2357-2367, Jun. 2017.

[6] K. J. Kim, M. D. Renzo, H. Liu, P. V. Orlik, and H. V. Poor, "Performance analysis of distributed single carrier systems with distributed cyclic delay diversity," IEEE Trans. Commun., vol. 65, no. 12, pp. 5514 - 5528, Dec. 2017.

[7] Y.-C. Liang, W. S. Leon, Y. Zeng, and C. Xu, "Design of cyclic delay deievsity for single carrier cyclic prefix (sccp) transmissions with blockiterative GDFE (BI-GDFE) receiver," IEEE Trans. Wireless Commun., 


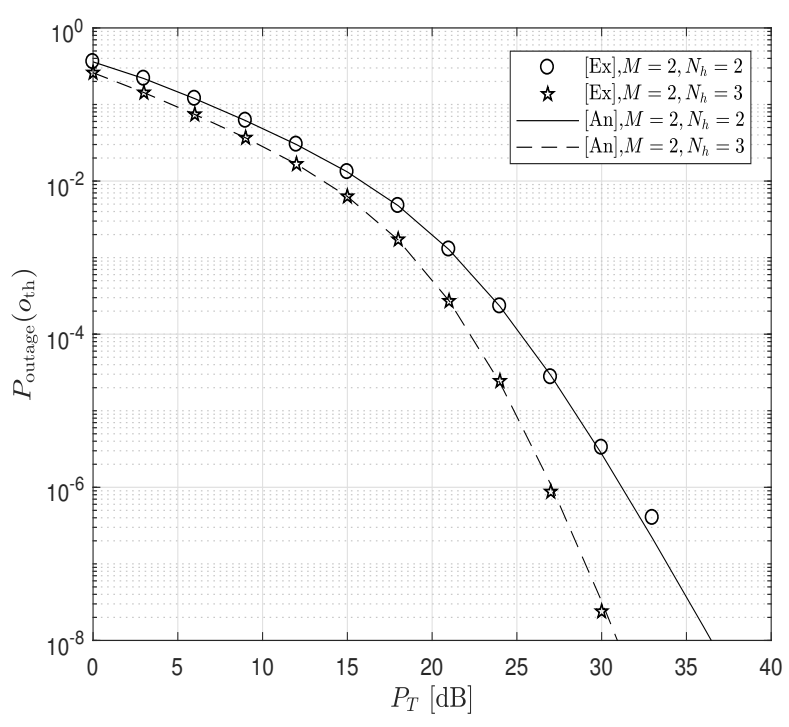

Fig. 2. Outage probability for various values of $N_{h}$.

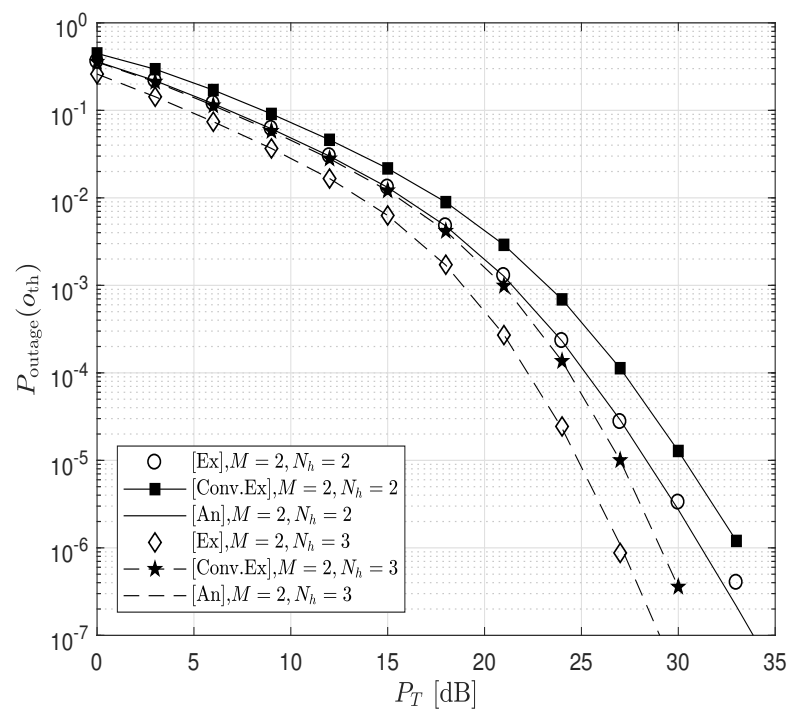

Fig. 3. Outage probability comparison with that of the existing work.

vol. 7, no. 2, pp. 677-684, Feb. 2008.

[8] A. H. Mehana and A. Nosratinia, "Single-carrier frequency-domain equalizer with multi-antenna transmit diversity," IEEE Trans. Wireless Commun., vol. 12, pp. 388-397, Jan. 2013.

[9] Q. Li, Q. Yan, K. C. Keh, K. H. Li, and Y. Hu, "A multi-relay-selection scheme with cyclic delay diversity," IEEE Commun. Lett., vol. 17, no. 2, pp. 349-352, Feb. 2013.

[10] U.-K. Kwon and G.-H. Im, "Cyclic delay diversity with frequency domain Turbo equalization for uplink fast fading channels," IEEE Commun. Lett., vol. 13, no. 3, pp. 184-186, Mar. 2009.

[11] K. J. Kim, T. A. Tsiftsis, and H. V. Poor, "Power allocation in cyclic prefixed single-carrier relaying systems," IEEE Trans. Wireless Commun., vol. 10, no. 7, pp. 2297-2305, Jul. 2011.

[12] K. J. Kim, T. Q. Duong, and H. V. Poor, "Outage probability of singlecarrier cooperative spectrum sharing systems with decode-and-forward relaying and selection combining," IEEE Trans. Wireless Commun., vol. 12 , no. 2 , pp. 806-817, Feb. 2013.

[13] K. J. Kim, L. Wang, T. Q. Duong, M. Elkashlan, and H. V. Poor, "Cognitive single-carrier systems: Joint impact of multiple licensed

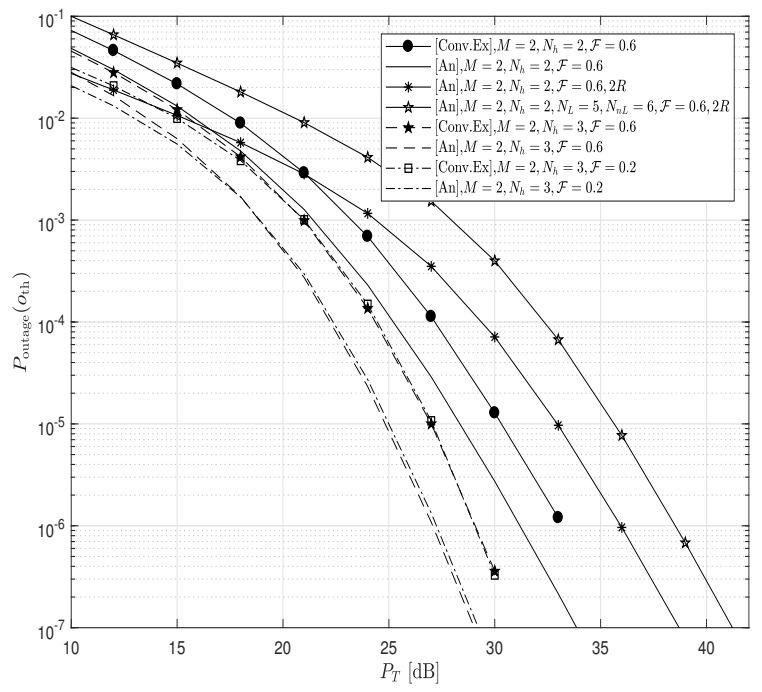

Fig. 4. Outage probability for various scenarios.

transceivers," IEEE Trans. Wireless Commun., vol. 13, no. 12, pp. 67416755, Dec. 2014

[14] X. Zhang and J. G. Andrew, "Downlink cellular network analysis with multi-slope path loss models," IEEE Trans. Commun., vol. 63, no. 5, pp. 1881-1894, May 2015.

[15] T. Bai, R. Vaze, and R. W. Heath, "Analysis of blockage effects on urban cellular networks," IEEE Trans. Wireless Commun., vol. 9, no. 13, pp. 5070-5083, Sep. 2014.

[16] M. Ding, P. Wang, D. Lopez-Perez, G. Mao, and Z. Lin, "Performance impact of LoS and NLoS transmissions in dense cellular networks," IEEE Trans. Wireless Commun., vol. 15, no. 3, pp. 2365-2380, Mar. 2016.

[17] 3GPP, TR 36.828 (V11.0.0), "Further enhancements to lte time division duplex (TDD) for downlink-uplink (DL-UL) interference management and traffic adaptation," Jun. 2012.

[18] C. M. Lo and W. H. Lam, "Performance of generalized selection combining for mobile radio communications with mixed cochannel interferers," IEEE Trans. Veh. Technol., vol. 51, no. 1, pp. 114-121, Jan. 2002.

[19] Y. Zeng and T. S. Ng, "Pilot cyclic prefixed single carrier communication: channel estimation and equalization," IEEE Signal Process. Lett., vol. 12 , no. 1 , pp. 56-59, Jan. 2005.

[20] F. Gao, A. Nallanathan, and C. Tellambura, "Blind channel estimation for cyclic-prefixed single-carrier systems by exploiting real symbol characteristics," IEEE Trans. Veh. Technol., vol. 56, no. 5, pp. 24872498, Sept. 2007.

[21] K. J. Kim, Y. Yue, R. A. Iltis, and J. D. Gibson, "A QRD-M/Kalman Filter-based detection and channel estimation algorithm for MIMOOFDM systems," IEEE Trans. Wireless Commun., vol. 4, pp. 710-721, Mar. 2005.

[22] A. P. Prudnikov, Y. A. Brychkov, and O. I. Marichev, Integral and Series. Vol. 3: More Special Functions, 3rd ed. London: Gordon and Breach, 1992.

[23] K. An, M. Lin, T. Liang, J. B. Wang, J. Wang, Y. Huang, and A. L. Swindlehurst, "Performance analysis of multi-antenna hybrid satelliteterrestrial relay networks in the presence of interference," IEEE Trans. Wireless Commun., vol. 63, no. 11, pp. 4390-4404, Nov 2015. 